\title{
The Potential of Blockchain Technology and Digital Economy of Bangladesh
}

\author{
Barna Bose ${ }^{\mathrm{a}}$, NaimurRahman ${ }^{\mathrm{b}}$ \\ abarna.bose@bup.edu.bd \\ bnaimur.rahman@bup.edu.bd
}

${ }^{a}$ Lecturer, Department of Economics, Bangladesh University of Professionals (BUP), Mirpur Cantonment, Dhaka-1216, Bangladesh.

'Lecturer, Department of Economics, Bangladesh University of Professionals (BUP), Mirpur Cantonment, Dhaka-1216, Bangladesh.

\begin{abstract}
:
Digital economy is relied on the trust of an online authorities. Complex transaction pattern made it difficult to confirm quick transfer of money between hands. Digital transactions provide a platform to make this complex nature of transactions easier for parties involved in online platform. of money Blockchain is a distributed ledger used to have a peer to peer online transaction system without the intervention of any centralized authorities. Blockchain technology facilitates the user with the maximum security during the transaction because the use of the cryptographic hash function by the system which generates a unique output that can not be changed if the transaction is successfully completed. Blockchain also contains a certain verification code for every transaction. It cannot be altered once transaction is completed. Individuals and organizations involved in various monetary interactions need to keep trust on each other. Blockchain technology also keep every record of the transactions being made. Bitcoin is the pioneer cryptocurrency used in blockchain technology is also a peer to peer digital currency.
\end{abstract}

Keyword: Digital currency, Blockchain, Cryptocurrency, Bitcoin, Distributed ledger; Cryptographic hash function, Cryptographic nonce, Crypto economy.

\section{Introduction:}

Unlike the past three industrial revolutions, we are at the brink of the fourth industrial revolution where technology is everything. The Internet is nothing but a tool for adding a new phase of communication and a decentralized system. More than twenty years of scientific research (van Rossum, 2018), there have been dramatic advances in Cryptography (Hileman, 2017). Blockchain, heavy use of cryptographic function which is fundamentally shifting how society operates to prevent double-spending, digital cash ("Blockchain," 2018) was conceptualized undine center server trusted system, before the advent of blockchain. Three decades later, bitcoin (Li, (2020).) entered the marketplace and replaced the idea of the center server.

One of the most important features of blockchain technology is its built-in transparency, which can profoundly use in identity management, government, and non-government record keeping as well as finance and banking. A country like Bangladesh, facing new challenges every day to emerge, needs a handful of care of wasted resources such as electricity and storage, scalability, usability, and privacy in every sector. Over the past few years, the banking system of Bangladesh become volatile (Sadekin, 2016) and the users seeking a new way to avoid using middleman, reducing transaction time and want to hold a digital identity. In this paper, we exemplified bitcoin as a modern currency, and tried to incorporate blockchain technology under mobile financial services along with its components and picturized it as a digital identity.

\section{Objectives:}

The blockchain technology itself and the issues of cryptography as such are not covered in this study. This paper aims to determine economic and financial reasons for the emergence of such a phenomenon as bitcoin and other cryptocurrencies, as well as to assess the degree of their integration into the economy of Bangladesh and the prospects for participation in the financial sector as full-fledged money of the digital economy of the future. In other words, 
depicts a comparative analysis of various forms of money that evolved throughout history, including cryptocurrencies in their present form. In a nutshell, the research objective can be portrayed as:

- To make an assessment and a quest for the development of motivational factors that should attract the government banking, industry, and another service sector to make a fully digitalized Bangladesh.

\section{Literature review:}

Historians note that ancient societies (Weatherford, 1998)found exchange goods with one another was often easier and safer than to go to battle for them. Scholars trace such exchange back into human prehistory, 10,000(Mauss, 2000) years or more ago. Humans initially considered barter as money, known as the direct exchange of goods and services. With the development of farming and later writing in Mesopotamia from the 8th millennium BC onward, the use of grains for trade became commonplace. Initially, in some societies, transactions were being made with tokens that resembled coins. The tokens(Griswold, 2003), labeled with an illustration of the object being sold, were meant to act as a reminder that an object has exchanged hands.". Around the 7th millennium BC in western and central Asia, companies established a means of trade based on the rich mineral resources of the region, extracting metals such as gold, copper, and tin. According to historians, coins were first crafted ("Coinage," n.d.)in the 5th century BC, in the ancient kingdom of Lydia, on the western coast of the region now known as Turkey. Electrum was the first coin that was made of a mixture of gold and silver. As widely seen today, the coins are not necessarily round and thin, but sometimes had irregular sizes and shapes, and were engraved on one side only. From Lydia, the use of electrum coins spread to Greek cities on the coast of Asia Minor, and then to the mainland of ancient Greece. The practice of counterfeiting followed suit almost as early as the rulers and nations began to produce coin currencies. Shave the edges of coins to gain material to produce new coins through forgery was the only utmost concern for an early age.

Cryptocurrencies have become the center of attraction for many economists (Böhme, 2015)especially for those who are more concerned about the transaction of money in online platforms (Janssen, 2020)The Blockchain network has been expanded through the massive online transaction demand by various business firms (Yeoh, 2017). Blockchain is considered as a platform where online transactions have been made with a more secured channel and with lower transaction costs for peer to peer transactions (Catalini and Gans, 2016). Blockchain enables the platform to facilitate transparent and valid transactions in the absence of intermediaries with the use of different technology (Cocco et, al., 2017). This often generates the expansion of efficiency of transactions, minimizing the transaction error, ensuring a quick transaction (McLean, 2016)There have been many questions raised about the ability of blockchain to generate a transaction system with the lowest cost and ability to store and processing of transaction history(Hileman, 2017). Bitcoin(Li, (2020). ) Cash holds a significant position in altcoin history, as it is one of the original bitcoins 's earliest and most popular hard forks. Because of the decentralized existence of digital currencies, drastic improvements need to be made to the code underlying the token or coin at hand due to consensus; the mechanism for this process generally varies but mostly depending on the cryptocurrency.

In this paper, we outline the potential benefits of blockchain technology along with various types of cryptocurrencies with their features, while also exploring the concrete challenges that this technology presents to law enforcement, how we contract. We also question whether this technology will enable the creation of advanced forms of digital rights management and, blockchain technology the last part was about Bangladesh's perspective under the digital phenomena to achieve self-sovereign identity ("SSL") and mobile financial service

\section{Methodology:}

This research mainly based on qualitative approaches. The information and the insights required in this research are the criteria for developing a good and effective digital economy of Bangladesh. In addition, all the information was collected from various journals, articles, tv news, and other online portals. As the idea of blockchain technology is very new and not being adopted by all the sectors of Bangladesh's economy, so that adopting strategies from techno prone countries and summarized in an effective way so that it creates a concern for all sectors of the economy.

\section{Bitcoin as a prominent modern cryptocurrency:}


Bitcoin is the first peer-to-peer (P2P) electronic case system which facilitates the payments to be sent online from one entity to another without the intervention of centralized authority (Nakamoto, 2008). Blockchain was the technology that enabled Bitcoin finally to remove the double-spending problem, thus, to emerge as the first online currency(Davidson, 2017). A trust is established through mass collaboration and clever code used on the Blockchain network system (Thomason, 2017)The Bitcoin system orders transactions by placing them in groups called blocks and then linking these blocks through what is called Blockchain (Nofer, 2017)

Bitcoin, a modern form of digital cryptocurrency, totally a new manifestation of traditional currency where no government legislation is needed. Bitcoin is a ledger, storing account information and their balance, which works like an online bank account that every user can access, receive, and transfer money. This is different from a traditional bank, wherein the information is controlled by the central authority. The ledger of Bitcoin is owned by everyone in the Bitcoin network (Chen, 2016)

\section{Blockchain technology: How it works?}

Blockchain is a database of transactions exchanged by everyone present in the network. With cryptocurrency, transaction records are stored as data blocks, which are chained together cryptographically. It is open to any node in the system and everyone can enter new entries (Chen, 2016) Blockchain is immutable; thus, each block is modified, it will always be regenerated once a block (McLean, 2016) is changed. Blockchain is a distributed ledger that is used to keep a record about any kind of online exchange of assets among parties those who are part of the blockchain with proper verification of the transaction. In a blockchain, each block relates to the previous blocks. So, any kind of transaction once done cannot be altered. An absence of any centralized authority makes it a more secure form of transaction platform. The use of blockchain technology confirms that each unit of value was transferred only once, solving the long-standing problem of double-spending.

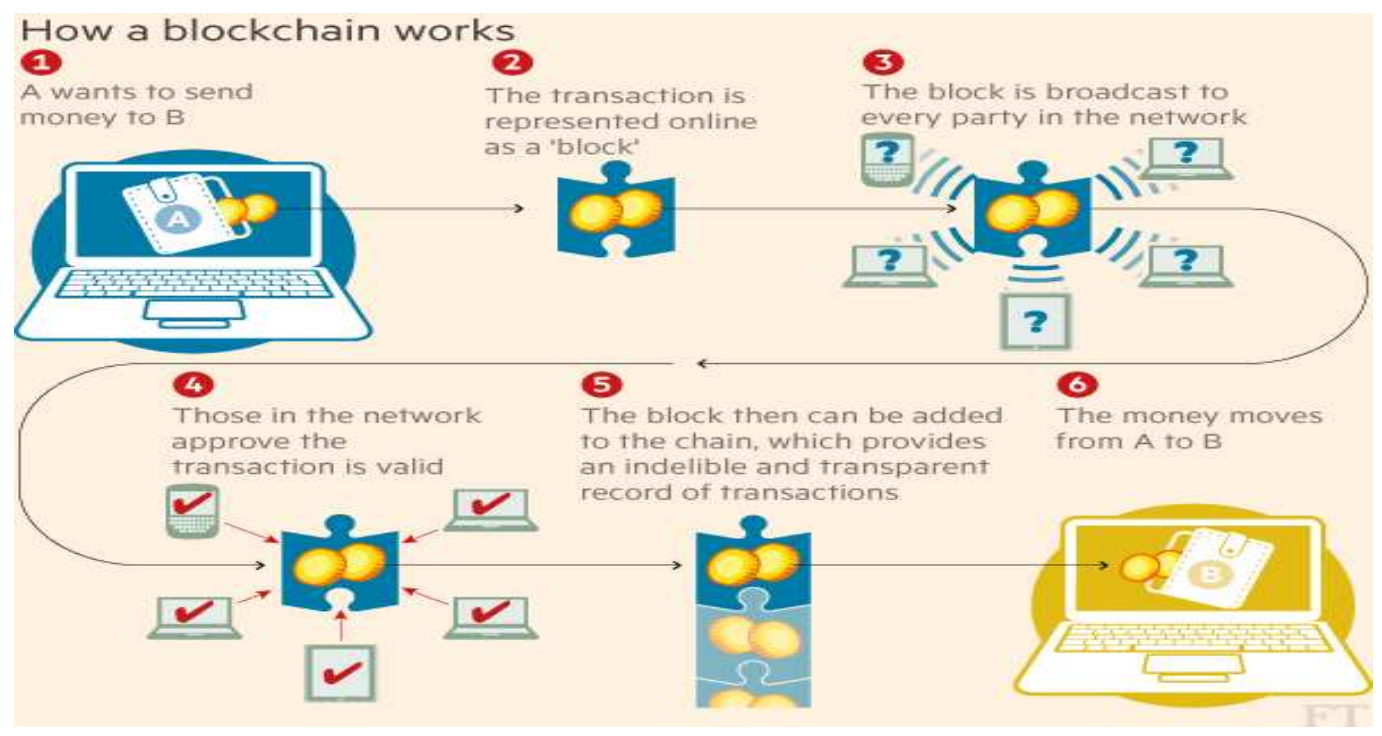

Figure 01: How a Blockchain technology works (Crosby et. al., 2016)

\subsection{Features of Blockchain technology:}

There are four key features of blockchain technology:

a) Ledger: A ledger is used to provide full information about the transaction and there is a minor possibility that the transactions \& value of the blockchain are overridden.

b) Secure: Transactions within the blockchain is cryptographically secured from unwanted guests which makes the ledger free from any forms of tampering the information regarding transactions. 
c) Shared: Blockchain technology maintain a decentralized system of transactions following the fact that multiple participants can share the ledger. Decentralized system of transactions makes blockchain more transparent.

d) Distributed: A blockchain technology uses a node to keep the transaction free from bad actors because blockchain can be distributed among multiple participants. Bad participants may not interrupt if the number of scales of nodes is higher (Yaga et. al., 2018).

\subsection{Blockchain components:}

\subsubsection{Cryptographic Hash Functions:}

Blockchain technology uses cryptographic hash functions for many operations. Cryptographic hash function to any data can be applied by using Hashing method. A relatively unique output is calculated by cryptographic Hash (called a message digest) for the input of nearly any size (e.g., a file, text, image). It facilitates individual agent in the blockchain to take input data, hash that data, and derive the same result (Yaga et. al., 2018).

\subsubsection{Cryptographic Nonce:}

A cryptographic nonce is an arbitrary number that is used only once and can be combined with data to produce different hash digests per nonce: hash $($ data + nonce $)=$ digest

\subsubsection{Transactions:}

A transaction generally portrays an interaction between two parties involved in a particular blockchain transaction. It represents a transfer of the cryptocurrency between the user of that blockchain network.

\subsubsection{Input:}

The inputs are usually a list of the digital assets to be transferred is called an input. Because the transaction data is a reference to past events, the digital assets remain unchanged. By proving access to the private key, the sender must also provide proof that they have access to the referenced inputs signing the transaction in the digital network (Yaga, (2018))

\subsubsection{Output:}

The outputs will be the recipients of the digital assets along with how much digital assets they will receive. Each output specifies the number of digital assets to be transferred by the sender to the new owner of the assets. (Yaga, (2018))

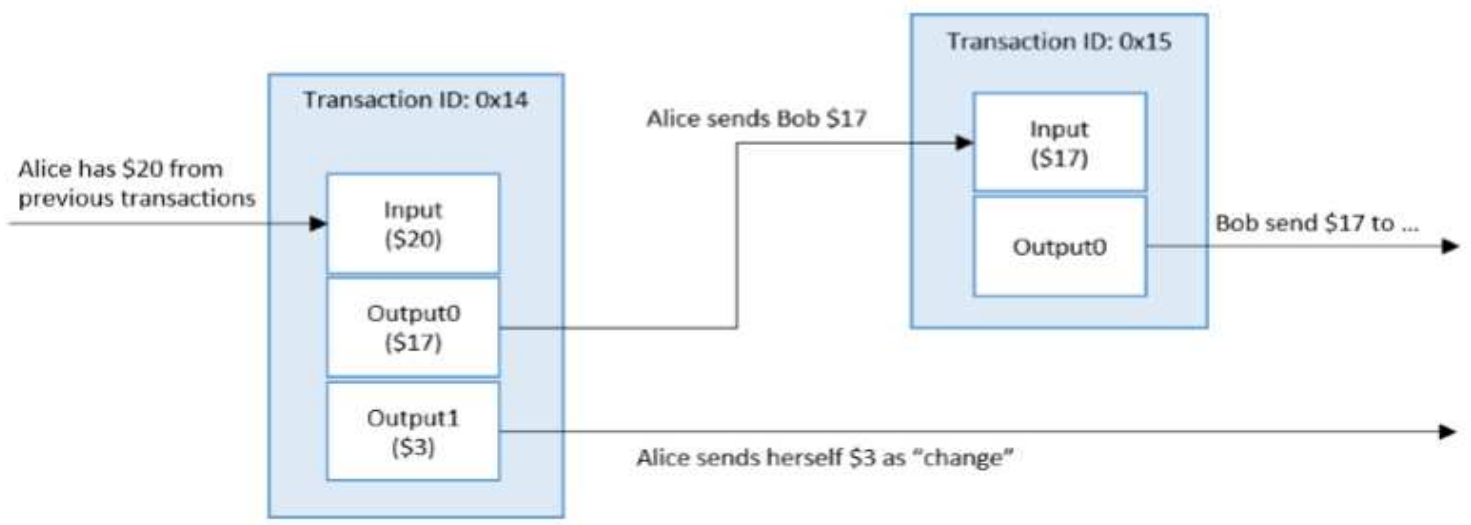

Figure 02: Cryptocurrency transaction process (yagaet. al., 2018) 


\section{Block chain: why it really matters?}

\subsection{Changing nature of money:}

Bitcoin offered an alternative trust base, questioning the traditionally presumed inseparability of money. In the analysis of crypto tokens, especially bitcoin, focus on its role as a means of exchange. Some experts raise the question of the volatility of bitcoin. Many of them argued that bitcoin is used widely accepted currency and considered it as doomed currency. Cryptocurrency as a medium of digital exchange is not strong enough to use as a strong store of value. But cryptocurrencies work as a strong source of a unit of measure of account. Cryptocurrency has introduced new ways to update the economic activity with the help of a crypto token helps to create a crypto economy.

\subsection{Crypto economy:}

The traditional way of sharing documents with collaborationis to send a Microsoft Word document to another recipient and ask them to revise it. The issue with this situation is that wait until the user gets a return copy till the user sees or makes any changes, since the user is locked out of editing it before the any person is finished with it. Instead of forwarding them on to each other, losing track of copies, and not being in touch with the other version, why can't all business documents be exchanged and not passed back and forth instead?So many types of legal contracts would be ideal for that kind of workflow.

\subsection{Smart contracts:}

With specified conditions, the ledger in the blockchain generates a coding to be executed during the transactions. This possibility has been found in the use of the Ethereum in 2018 where it has been used as an open-source network. Various up-gradation has been done to promote the usefulness of blockchain. Various smart can be programmed to perform simple online transactions.

\subsection{The sharing economy:}

The sharing economy has been promoted worldwide with an amazing success like UBER. In Uber, people who are willing to hail a cab must rely on an intermediary like Uber and Pathao. Blockchain technology widens the door of a peer to peer direct connection for any kind of online transactions through eliminating the role of the centralized authority.

\subsection{Supply chain auditing:}

An ethical point of view to a day has become the key concern of the consumers as they seek a transparent way of transactions. The distributed ledger used in the blockchain provides an easy way to certify the background of the transaction being a genuine transaction. Blockchain plays a huge role to ensure a transparent way of transactions of a diamond.

\subsection{Identity management:}

Developing a digital identity is not an easy task to accomplished. Establishing a digital identity requires the cooperation between private and public entities. Online interaction also requires having a secure online identity. Blockchain technology provides that platform to build a share economy through conducting online transactions. SSL certificates are used to conduct E-commerce in an online platform where Netki provides the channel to create an SSL for blockchain.

\subsection{Data management:}

Blockchain is considered as a trusted source of keeping data related to online transactions. Both buyer and seller engage themselves in online transactions through blockchain can be able to secure their transactions in the absence of a centralized authority.Today people can use social media sites such as Facebook for free, in exchange for their personal data. 


\section{How can blockchain be used to give Bangladesh a digital identity?}

The World Economic Forum has called blockchain a "mega-trend" and the United Kingdom's Department for International Development's innovation chief, Tamara Giltsoff, called blockchain the next tech gamechanger(Thomason, 2017). Blockchain technology using as a decentralized tool for both the nation and individual, often called a self-sovereign identity (SSI) ("Self-sovereign identity," 2018).

With high institutional value and holding the motto of "SDG 9: Build a resilient infrastructure, promote inclusive and sustainable industrialization and foster innovation" Bangladesh is marching forward to growing up as a digitalized country and look over quite a few things, E-KYC- carryout by maximum financial institutions for the customers, Reputation system- rating and recommendation through social media as well as online-based pension system (Sami Ahmed, 05 Jan 2020)

With over 160M people in Bangladesh, Despite the notable expansion of financial institutions and services over time, a recent World Bank study has claimed that almost half of Bangladeshis aged 15 or above does not have a bank or mobile money account. The latest edition of Findex showed that in 2014 only three percent of adult population owned mobile money account which has increased to 21 percent by 2017. Due to the lack of a verifiable digital identity, these services remain somewhat stagnant. A recent report on Computerworld published that Blockchain allows with a simple smartphone to create an identity on the blockchain by using digital data, whether it's behavioral data, commercial data, health data, browsing data or something, who have not previously had a digital identity to use a $\$ 10$ smartphone from their home comfort.(Bryzek, 2018)

By using blockchain existing land registration process can be replaced with clarity, consisting of third-party transaction in a block (buyer, seller, and the land register office) in a faster time, without corruption and tempering of documents by fake ownership. Using blockchain-based tourism Saint Martin can be well regulated, maintain payment settlement, and visitor control in a central control mechanism by the national tourism board of Bangladesh to protect this precious coral island. Moreover, using blockchain in public services like education, transportation, port - shipping, land registration office, passport office, and audit firm government can ensure maximum utilization of minimum resources by reducing corruption and redtops. Thus, a "good" digital identity(Dakota Gruener, 2019) for the people of Bangladesh who are under seasonal migration, leaving their ruined homes and disappearing occupations behind for weeks or months on end as they search for work in large cities could be better in needful ways.

\subsection{Bangladesh Receptive to Mobile Financial Services:}

Payment is an important part of every business across the world, financial technology (fintech) companies are bringing about these positive changes and overhauling the payment industry with the help of technology. By the end of 2020 , digital technology will integrate at least 20 billion devices along with more than 5 billion people and businesses (Hossain, 2020).

BKash ("bKash," 2018)is one of the earliest and dominant mobile financial services entered 2011 into the Bangladesh market to provide a means to electronically transfer money without having an existing bank account. By using a mobile number and national identity card, any Bangladeshi citizen can open it. By 2017, over 22\% of adults use bKash with around 4.5M transactions each day, as of May 2019 its active user is around 3.1 crore.

Digital Financial Service (DFS) connecting the dots between the physical and virtual elements of business through the integration of man and machine with the medium of finance (Hossain, 2020). Bangladesh is already making a position at Mobile Financial Services (MFS) (Morawczynski, 2009) Provided the lowest transaction cost in Bangladesh Along with Bkash, Nagad, rocket, and various merger companies made mobile cash transactions as a part of daily rituals. Bangladesh already boasts $117 \mathrm{M}$ smartphone subscriptions in the past few years. A report from New Delhi said, in 2019, The average cost of a smartphone in Bangladesh is \$99 with a 4.1 percent annual growth, with 29.6 million units shipping can consider as another milestone. In a nutshell, blockchain technology opens a new window for FinTech industry of Bangladesh.

\section{Concluding remarks:}

The choice between traditional currency and modern currency is not trivial. While this question has been discussed before to the best of knowledge, we provide in this article the first structured methodology to decide which technological solution is the most appropriate depending on which application scenario. We applied our methodology to find the potentials and why people should trust this kind of trustable online transaction methods to ignore real-life 
shoe-leather costs. We conclude that depending on the application scenario, there are indeed valid use cases for each monetary system and centralized databases that need to be determined carefully. We consider a developing country like Bangladesh which has an enormous possibility to be digitalized along with MFS and SSI.

\section{References}

Böhme, R. C. (2015). Bitcoin: Economics, technology, and governance. Journal of economic Perspectives, 29(2), 213-38.

Bryzek, P. (2018, February 26). Blockchain Positioned to Save Bangladesh. medium.

Chen, Z. Z. (2016). Smartphone inertial sensor-based indoor localization and tracking with iBeacon corrections. . IEEE Transactions on Industrial Informatics, 12(4), , 1540-1549.

Dakota Gruener, A. C. (2019). Opinion: The case for 'good' digital identity. Devex.

Davidson, S. D. (2017). Blockchains and the economic institutions of capitalism. Journal of Institutional Economics, 14(4).

Griswold, C. W. (2003). smart token. Washington, DC: U.S. Patent and Trademark Office: . U.S. Patent No. $6,629,591$.

Hileman, G. \&. (2017). Global cryptocurrency benchmarking study. Cambridge Centre for Alternative Finance, 33, $33-113$.

Hossain, R. (2020). FINTECH - in the context of Bangladesh. Financial express.

Janssen, M. W. (2020). A framework for analysing blockchain technology adoption: Integrating institutional, market and technical factors. . International Journal of Information Management, 50, 302-309.

Li, X. J. ( (2020). ). A survey on the security of blockchain systems. Future Generation Computer Systems,. 107, $841-853$

McLean, J. (2016). Banking on blockchain: charting the progress of distributed ledger technology in financial services. . A Finextra white paper produced in associate with IBM.

Nakamoto, S. (2008). Bitcoin: A peer-to-peer electronic cash system.

Nofer, M. G. (2017). Blockchains and the economic institutions of capitalism. Blockchain. Business \& Information Systems Engineering, 59(3), ., 183-187.

Sami Ahmed, D. M. ( 05 Jan 2020). National Blockchain Strategy: Bangladesh. Bangladesh: ICT division.

Thomason, J. (2017). Opinion: 7 ways to use blockchain for international development. devex.

Yaga, D. M. ( (2018)). Blockchain Technology Overview. . National Institute of Standards and Technology, NISTIR 8202 . Retrieved from Recuperado de: https://nvlpubs. nist. gov/nistpubs/ir/2018/NIST. IR, 8202

Yeoh, P. (2017). Regulatory issues in blockchain technology. Journal of Financial Regulation and Compliance. 